This item was submitted to Loughborough's Research Repository by the author.

Items in Figshare are protected by copyright, with all rights reserved, unless otherwise indicated.

\title{
Investigating optimal technique in a noisy environment: application to the upstart on uneven bars
}

PLEASE CITE THE PUBLISHED VERSION

http://dx.doi.org/10.1016/j.humov.2012.11.004

PUBLISHER

(C) Elsevier

VERSION

AM (Accepted Manuscript)

LICENCE

CC BY-NC-ND 4.0

\section{REPOSITORY RECORD}

Hiley, Michael J., and Maurice R. Yeadon. 2019. "Investigating Optimal Technique in a Noisy Environment: Application to the Upstart on Uneven Bars". figshare. https://hdl.handle.net/2134/12288. 
This item was submitted to Loughborough's Institutional Repository (https://dspace.lboro.ac.uk/) by the author and is made available under the following Creative Commons Licence conditions.

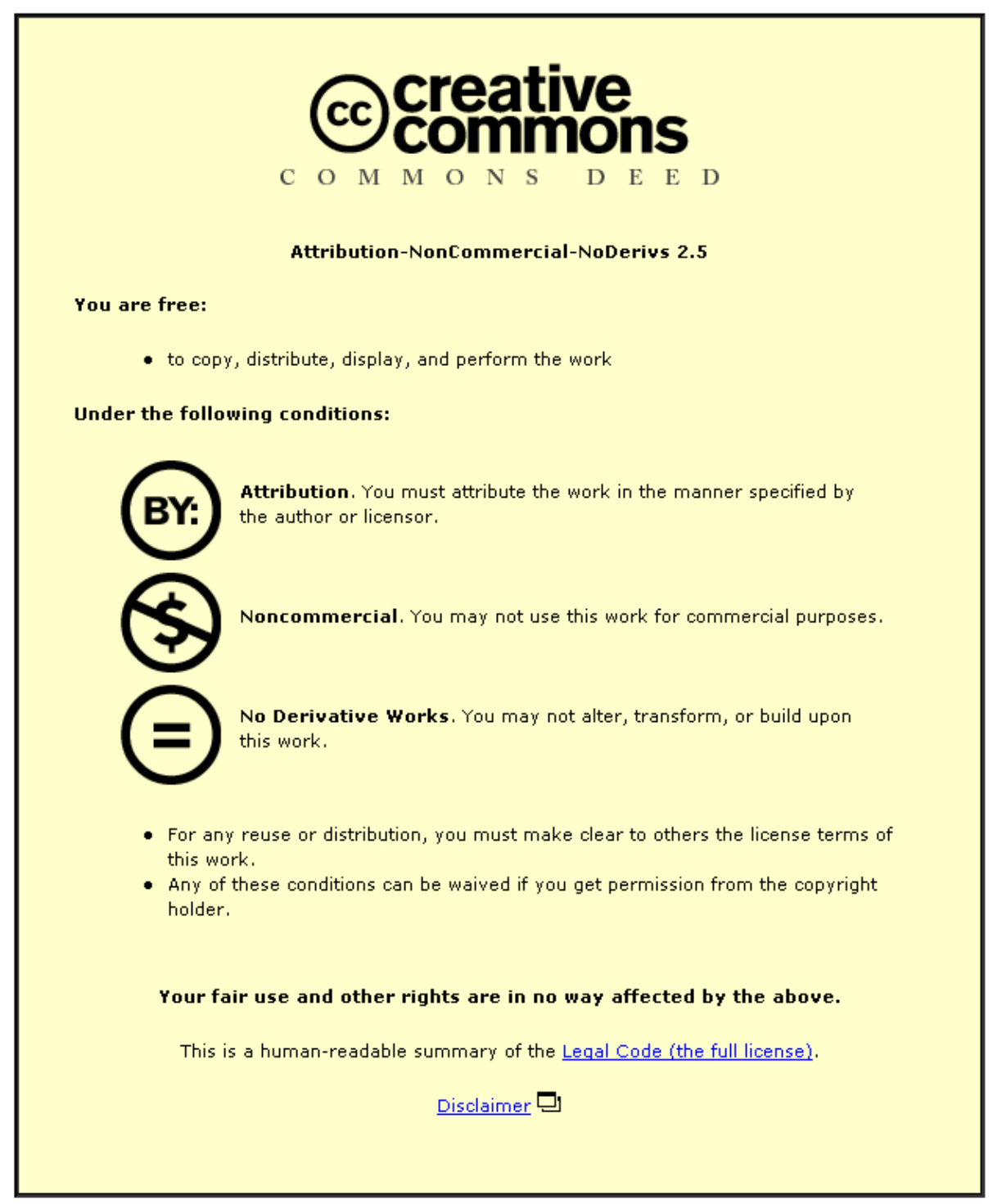

For the full text of this licence, please go to: http://creativecommons.org/licenses/by-nc-nd/2.5/ 
Investigating optimal technique in a noisy environment: application to the upstart on uneven bars

\author{
${ }^{1}$ Michael J Hiley and ${ }^{1}$ Maurice R Yeadon
}

${ }^{1}$ School of Sport, Exercise \& Health Sciences, Loughborough University, Loughborough, LE11 3TU, UK

\begin{abstract}
The upstart is a fundamental skill in gymnastics which is used to transfer a gymnast from a swing beneath the bar to a position above the bar. The aim of this study was to optimise the technique in the upstart on the uneven bars in order to determine the underlying control strategy used by gymnasts. A previous attempt based on minimising joint torque had failed to find a satisfactory solution without forcing the joint angle histories to pass through a "via-point" (Yamasaki et al., 2010). Using a computer simulation model of a gymnast and bar, the technique (joint angle histories) used in the upstart was optimised under three different criteria: minimising joint torque, minimising joint torque change and maximising success in the presence of movement variability. The third optimisation introduced "noise" into the joint angle time histories based on measurements of kinematic variability. All three optimisations were started from the technique used by a gymnast competing in an Olympic Games uneven bars final. Root mean squared (RMS) differences between the recorded and optimal joint angle time histories were computed. The two optimisations based on minimising joint torque diverged from the gymnast's technique. However, the technique based on maximising the number of successful performances in a noisy environment remained close to the gymnast's technique. It is concluded that the underlying strategy used in the upstart is not based on minimisation of joint torque; rather, it is based on ensuring success in the task despite the inherent variability in technique. Gymnasts develop techniques that are able to cope with the level of kinematic variability present in their movements.
\end{abstract}

\title{
1. INTRODUCTION
}

The upstart is a fundamental skill in artistic gymnastics where it is used to transfer the gymnast from a swing beneath the bar to a position above the bar (Figure 1). The skill is typically used in women's artistic gymnastics when moving between the uneven bars, and after release and regrasp skills where the gymnast has insufficient amplitude of swing to move directly above the bar. The upstart comprises a swinging phase (Figure 1 A-B) and a "kip" phase (Figure $1 \mathrm{C}-\mathrm{E}$ ). In order to receive no deductions from the judges (FIG, 2009) all phases should be performed with straight arms and legs (Figure 1).

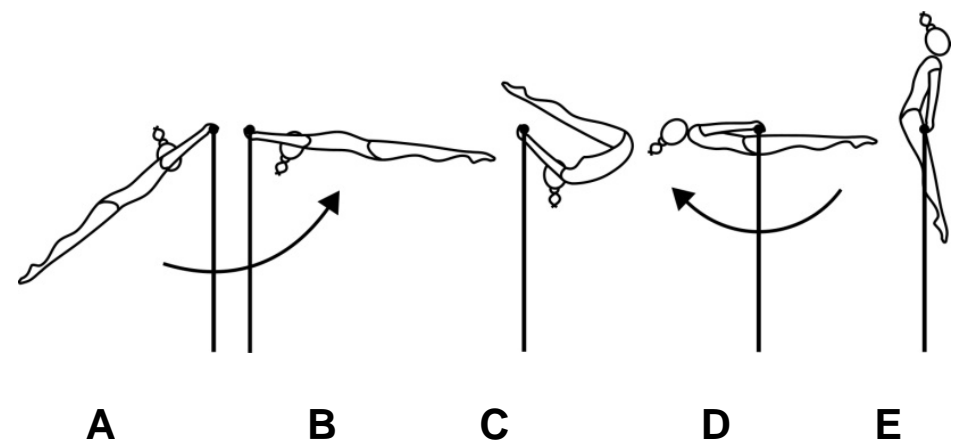

Figure 1. The upstart (adapted from the FIG Code of Points, 2009). 
Yamasaki, Gotoh and Xin (2010) presented a simulation model for swinging on bars and investigated the underlying control strategy of the upstart by optimising technique under various criteria. The optimal solutions (techniques) were compared to recorded gymnast performances and the criterion that compared most favourably was deemed to be the likely control strategy. The optimisation criteria were based on minimising angle jerk, joint torque change and effort. None of the criteria could reproduce the technique used by the gymnasts without forcing the model through an arbitrary via-point at which the shoulder and hip angles were matched to the recorded gymnast performance. It should be concluded that none of the optimisation criteria could account for the techniques used by gymnasts. There must be some other criterion that can explain the gymnasts' technique or some important aspect of human movement that has been overlooked.

In movements where a gymnast must exert near maximal effort to achieve the performance outcome, technique may or may not be based on minimising joint torque. For example, in the undersomersault to handstand on parallel bars beginners often adopt a technique similar to that used in the clear circle to handstand on high bar as it is less demanding in terms of strength compared to the more commonly performed stoop stalder technique adopted by senior gymnasts (Davis, 2005). The clear circle technique was predicted by minimising peak joint torque at the shoulder and hip using a simulation model (Hiley and Yeadon, 2012a). However in order to produce the stoop stalder technique, an optimisation criterion based on performance requirements (vertical path of the mass centre) was required and resulted in a technique that used close to maximal effort for the gymnasts studied. The underlying control strategy for the stoop stalder undersomersault technique is therefore not based on minimising joint torque.

Timing the actions (flexions and extensions) at the shoulder and hip can be a critical factor in the successful performance of the upstart, particularly in the early stages of learning. Broderick and Newell (1999) showed that in a task that required precise timing for success, skilled performers demonstrated lower levels of movement variability. When a gymnast performs the same skill a number of times it might be expected that within each attempt there will be some variability in the technique used, i.e. variability in the timings and angles of the shoulder and hip angle time histories (Newell and Corcos, 1993). A gymnast's technique should therefore be robust so that it can produce similar results (e.g. successful completion of the upstart) despite the variability present. Hiley and Yeadon (2012b) calculated the level of kinematic variability in the start and end time of flexions and extensions at the shoulder and hip and in the magnitude of the angle changes during giant circles before a Tkatchev release and regrasp skill in 10 repeated trials. The average standard deviations in the temporal and spatial measures were 10 $\mathrm{ms}$ and $2.3^{\circ}$ respectively for a senior elite male gymnast. The measures of kinematic variability (Hiley and Yeadon, 2012b) would have included components of noise and any feedback corrections made by the gymnast (Cohen and Sternad, 2009; Harris and Wolpert, 1998). When the gymnast's giant circle technique was optimised, realistic solutions were obtained only when the variability present in the actual performances was included.

The aim of the present study is to optimise the technique in the upstart under various criteria (i.e. minimising joint torque, minimising torque change and maximising success) in order to determine which best describes the strategy used by gymnasts. The study will incorporate the level of kinematic variability present in elite gymnasts' technique in the optimisation to investigate whether the strategy is determined by the 
need to be successful at the task rather than the need to minimise joint torque. All optimisations will be carried out using a computer simulation model.

\section{METHODS}

Subsections in Methods describe the protocol used to determine the control strategy used by gymnasts in the upstart on the uneven bars. Data collection was carried out in which an elite performance of an upstart from the Olympic Games was recorded and the data processed for subsequent use with a computer simulation model of swinging on bars. The simulation model was used with various optimisation criteria to determine the underlying control strategy for the upstart.

\subsection{Data collection}

All eight uneven bars routines from the Sydney 2000 Olympic Games women's artistic gymnastics apparatus final were recorded using two digital video cameras (Sony Digital Handycam DCR-VX1000E), operating at $50 \mathrm{~Hz}$ with shutter speeds of 1/600 s. The two cameras were located $8 \mathrm{~m}$ above the landing surface and $30 \mathrm{~m}$ and $37 \mathrm{~m}$ from the bars with a camera axis intersection angle of $66^{\circ}$. Prior to the start of competition a calibration structure comprising 26 spheres of diameter $0.10 \mathrm{~m}$ spanning a volume measuring $3 \mathrm{~m} \times 4.5 \mathrm{~m} \times 4 \mathrm{~m}$ was positioned so as to include the whole of a routine and was video recorded for use in the calibration procedure. Although the upstart was recorded in 2000 it was performed by an elite gymnast using a fundamental technique that has remained unchanged for many years.

The centres of the calibration spheres were digitised in five video fields from both camera views. An international standard (FIG brevet) judge selected the best upstart performed on the upper bar from the eight recorded routines. In each of the movement fields the centre of the hand and elbow, shoulder, hip, knee and ankle joint centres and toes on each side of the body were digitised along with the centre of the gymnast's head and the centre of the bar midway between the gymnast's hands. The data obtained from digitising the images of the calibration spheres and their known locations were used to calculate the 11 Direct Linear Transformation parameters for each camera (Abdel-Aziz and Karara, 1971). The two sets of digitised movement data were synchronised using the method of Yeadon and King (1999). Synchronised digitised coordinate data from each camera view along with the camera parameters were used to reconstruct the threedimensional locations of the body landmarks using the Direct Linear Transformation. Joint angles for the left and right sides were averaged and quintic splines (Wood and Jennings, 1979) were used to fit the orientation and joint angle time histories so that derivatives could be obtained (Yeadon, 1990a) for input to a planar simulation model.

A set of anthropometric measurements of an "average" elite female gymnast was obtained as the mean measurements taken from eight Romanian international gymnasts. The 95 mean values were then scaled to the Olympic competitor using segment lengths and widths obtained from the video digitisation and inertia parameters were calculated using the inertia model of Yeadon (1990b).

\subsection{Simulation model}

A four segment planar model of a gymnast comprising arm, torso, thigh and lower leg segments was used to simulate the movement around the bar (Hiley and Yeadon, 2003). The bar and the gymnast's shoulder structure were modelled as damped linear 
springs (Figure 2). The spring at the shoulder represented the increase in length of the gymnast between the wrist and the hip (i.e. not just the stretch at the shoulder joint). In addition to the shoulder spring, there was a parameter that governed the extent to which the torso segment lengthened (scapular elevation) as the shoulder elevation angle was increased (Begon, Wieber and Yeadon, 2009).

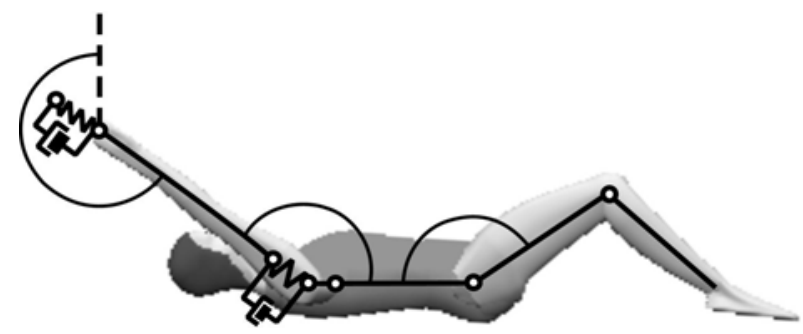

Figure 2. The four segment gymnast - bar simulation model with damped springs representing bar and shoulder elasticity.

Input to the simulation model comprised the segmental inertia parameters, the stiffness and damping coefficients of the bar and shoulder springs, the initial displacement and velocity of the bar, the initial angular velocity of the arm, the initial orientation of the arm and the joint angle time histories of the shoulder, hip and knee in the form of quintic splines (Wood and Jennings, 1979). Output from the model included the time histories of the horizontal and vertical bar displacements and the arm angle $\phi$ (the angle from the vertical of the line joining the bar to the shoulder centre). The equations of motion were derived using Newton's Second Law and by taking moments about the unloaded bar position and the segment mass centres. To obtain model parameters which could not be calculated directly (e.g. spring stiffness and damping coefficients) a matching procedure was carried out as described in Hiley and Yeadon (2007). A simulation was run with the matched parameters to assess the closeness of fit to the recorded data. Root mean squared differences were calculated between the time histories of the simulated and recorded arm angle and bar displacements (since these describe the whole body movement).

\subsection{Optimisation}

Three optimisations were carried out to determine the control strategy used to perform the upstart. All simulations performed within the optimisations started with an arm angle of $160^{\circ}$ and the initial conditions from the recorded performance. All simulations ended once the arm angle corresponded to the vertical support position above the bar (Figure 3a). A set of parameters which defined turning points in the joint angle time histories was used to manipulate the quintic splines driving the simulation. The turning points for the shoulder and hip are identified in Figure 3a. A data set spanning each joint angle time history obtained from the video analysis was transformed between each turning point in both the time and angle dimensions (Figure $3 \mathrm{~b}$ and equations $1 \& 2)$. 


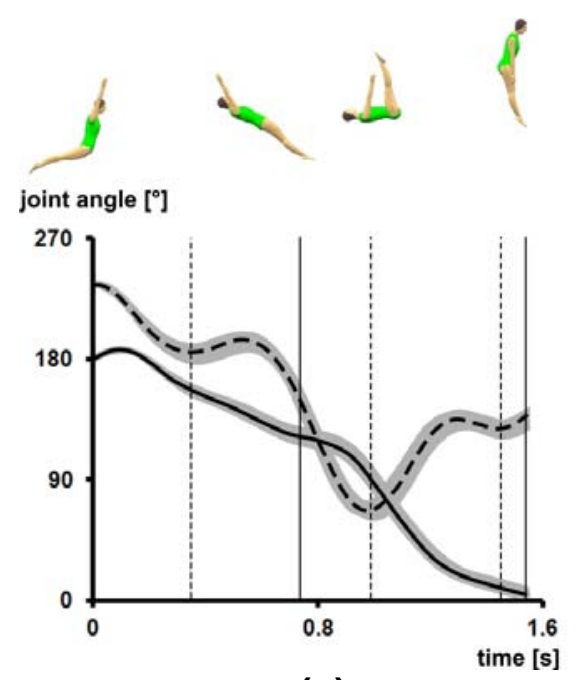

(a)

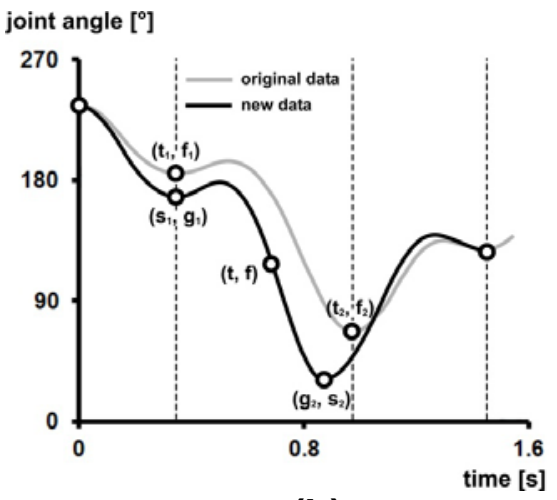

(b)

Figure 3. The joint angle history (a) at the shoulder and hip during the upstart with the turning points indicated (solid line for shoulder and dashed line for hip) and the envelope generated from 20 perturbed joint angle histories shown in grey and (b) the method of transforming the angle data between two turning points.

Any point $(t, f)$ in the new data set (Figure $3 b)$ can be calculated using equations 1 and 2 .

$$
\begin{aligned}
& t \rightarrow s_{1}+\left(\frac{t-t_{1}}{t_{2}-t_{1}}\right) \cdot\left(s_{2}-s_{1}\right) \\
& f \rightarrow g_{1}+\left(\frac{f-f_{1}}{f_{2}-f_{1}}\right) \cdot\left(g_{2}-g_{1}\right)
\end{aligned}
$$

where $t$ and $s$ are time parameters and $f$ and $g$ are angle parameters. An interpolating quintic spline was then fit to the new data set to allow the calculation of first and second derivative data which is required to drive the simulation model. The quintic spline method was used since it allowed more rapid changes between flexion and extension at a joint compared to the piecewise quintic function method previously described by Hiley and Yeadon (2003). Throughout the simulations the arms and legs were constrained to stay straight in keeping with good execution. The simulation model was incorporated within a genetic optimisation algorithm (Carroll, 2001) which manipulated the parameters defining the joint angle time histories at the shoulder and hip. The parameters comprised the time and angle at each of the turning points. For all optimisations the bounds for each parameter were initially set to either $\pm 30^{\circ}$ or $\pm 0.03 \mathrm{~s}$ of the values obtained in the matching simulation, depending on whether it was an angle or time parameter respectively. If an optimal solution reached any of the bounds the optimisation was repeated using the current solution as a starting point and a new set of bounds as described above. This was repeated until the optimal solution reached none of the bounds. All joint angle histories were constrained using joint torque measurements determined from an isovelocity dynamometer for a male National Team gymnast and by fitting a function which expressed maximum voluntary torque in terms of joint angle and angular velocity (Forrester, Yeadon, King and Pain, 2011). From the matching simulation 
it was found that the female gymnast worked within these maximal joint torques. The joint torque functions were then scaled based on the maximum percentage of the male maximal joint torque used in the matching simulation (King, Kong and Yeadon, 2009). If a simulation produced a joint torque within an optimisation that exceeded the scaled maximal value the simulation was given a penalty. In addition joint angle time histories which resulted in the bar passing through the leg segment were given a penalty score. In all three optimisations the recommended optimisation algorithm tuning (Carroll, 2001) was used.

In the first optimisation the criterion was based on minimising the shoulder and hip joint torque throughout the simulation (equation 3).

$$
F_{1}=\frac{1}{n} \sum_{i=0}^{n}\left(\left|T_{1}\right|+\left|T_{2}\right|\right)
$$

where $T_{1}$ and $T_{2}$ are the joint torques at the shoulder and hip respectively and $i$ is the simulation integration step counter. In order to determine how the optimal simulations compared to the recorded performance, penalties were used to encourage the model to reach the same configuration (shoulder and hip angles) at the end of the simulation. Since the aim was to determine the strategy used by the gymnast a further penalty encouraged the simulation to have the same duration as the recorded performance. These penalties would also permit comparisons with results from the literature (Yamasaki et al., 2010).

In the second optimisation the criterion was based on minimising the torque change at the shoulder and hip joints (equation 4) along with the same penalties on configuration and duration as the first optimisation.

$$
F_{2}=\frac{1}{n} \sum_{i=0}^{n}\left(\left|T_{1_{i}}-T_{1_{i+1}}\right|+\left|T_{2_{i}}-T_{2_{i+1}}\right|\right)
$$

In the third optimisation the criterion was based on maximising the number of successful upstarts despite operating in a "noisy" environment. For each set of joint angle time history parameters produced by the genetic optimisation algorithm (Carroll, 2001), an initial simulation was run with no perturbations added to the parameters. The simulation was given a penalty to encourage the same duration as the recorded performance (i.e. as in the first and second optimisations). Subsequently 999 randomly perturbed simulations were based on the same set of parameters, where perturbations were added to the time and angle parameters of the joint angle histories with a specified standard deviation (i.e. the average standard deviations of $10 \mathrm{~ms}$ and $2.3^{\circ}$ from Hiley and Yeadon, 2012b) using a random number generator and the method of Hiley and Yeadon (2012b). In the present study the kinematic variability introduced with the perturbations represented the noise component of the movement variability. The envelope generated from 20 perturbed joint angle histories is shown in grey on Figure 3a. Simulations were given a score of 1 for a successful upstart and 0 for an unsuccessful upstart. A successful simulation was one where the model reached the correct orientation with an appropriate configuration, that is, with the shoulder and hip angles within $10^{\circ}$ of the recorded performance (to allow for the addition of noise to the angle parameters). In the third optimisation exceeding the joint torque limits constituted an unsuccessful upstart. 
The root mean squared difference between the recorded and optimal joint angle time histories were calculated for both the shoulder and hip angles for each of the three solutions.

\section{RESULTS}

The matching simulation was able to match the arm angle to $4^{\circ}$ and the bar displacements to $0.008 \mathrm{~m}$ (root mean squared differences) of the recorded performance (Figure 4).

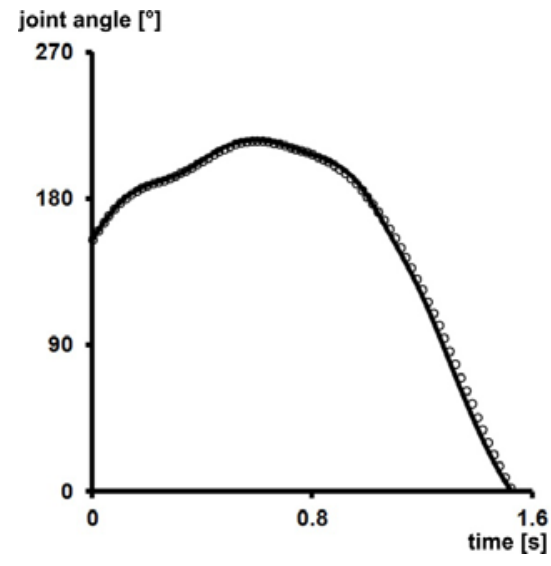

(a)

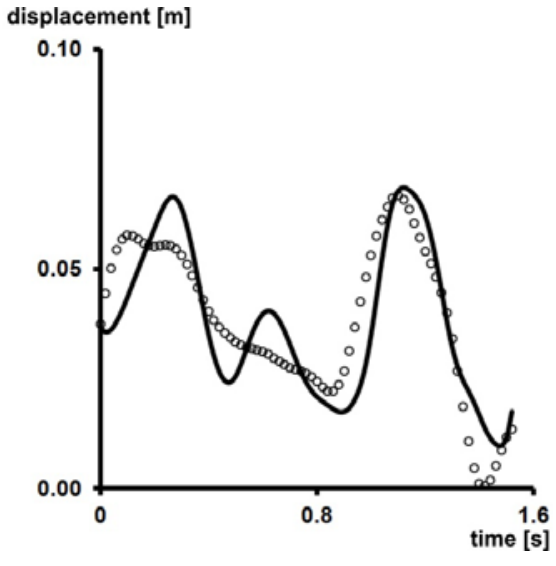

(b)

Figure 4. Matching simulation (solid lines) compared with recorded performances (circles) for: (a) arm angle and (b) bar displacement.

None of the optimal solutions obtained a penalty for end configuration, duration or exceeding joint torques. In the first and second optimisations the criterion was based on minimising joint torque and joint torque change. In the third optimisation the criterion was based on maximising the number of successful performances despite perturbations to the joint angle time histories. The first and second optimisations converged. However, due to the nature of the third optimisation convergence did not occur. Instead the final generations each produced $100 \%$ success but did not settle on a single set of joint angle parameters. There existed a region which spanned, on average, $3^{\circ}$ and $9 \mathrm{~ms}$ within which $100 \%$ success was obtained.

The angle and torque time histories of the first and second optimisations deviated from those of the matching simulation (Figures $5 \& 6$ and Table 1 ) whereas the angle and torque histories of the third optimisation compare well with the matching simulation (Figures $5 \& 6$ and Table 1).

Table 1. Root mean squared differences between the joint angle time histories of the recorded trial

\begin{tabular}{ccc}
\hline Optimisation criterion & $\begin{array}{c}\text { Shoulder } \\
{\left[{ }^{\circ}\right]}\end{array}$ & $\begin{array}{c}\text { Hip } \\
{\left[{ }^{\circ}\right]}\end{array}$ \\
\hline Minimise joint torque & $4^{\circ}$ & $51^{\circ}$ \\
Minimise torque change & $9^{\circ}$ & $18^{\circ}$ \\
Maximise success & $2^{\circ}$ & $5^{\circ}$ \\
\hline
\end{tabular}


(a)
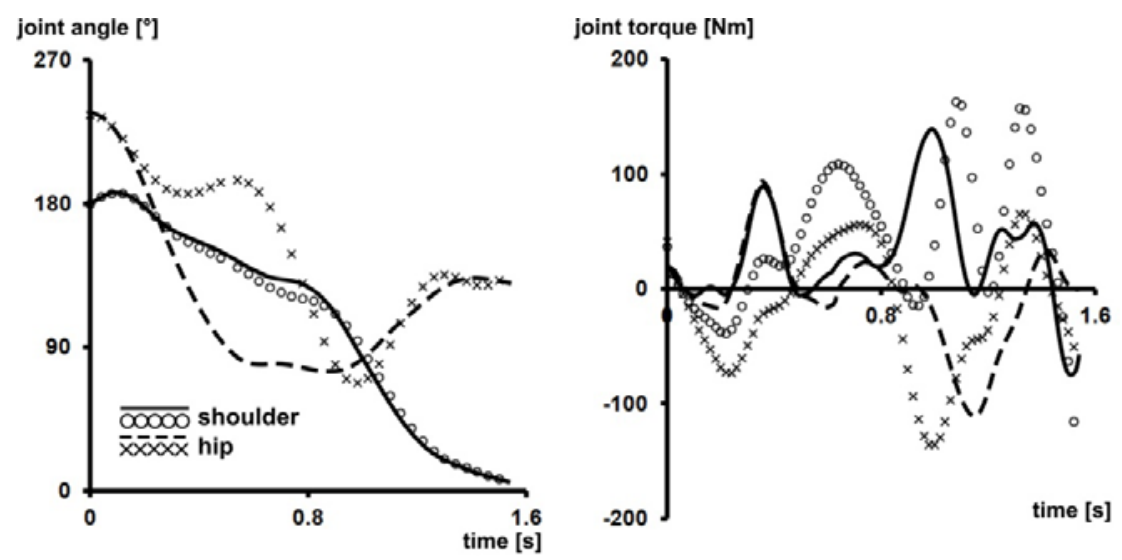

(b)
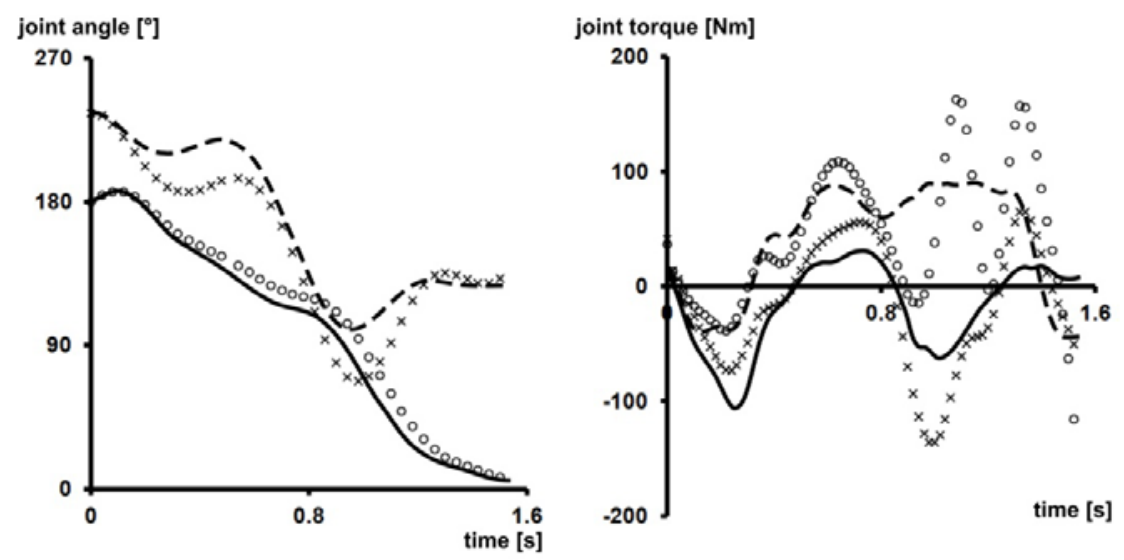

(c)
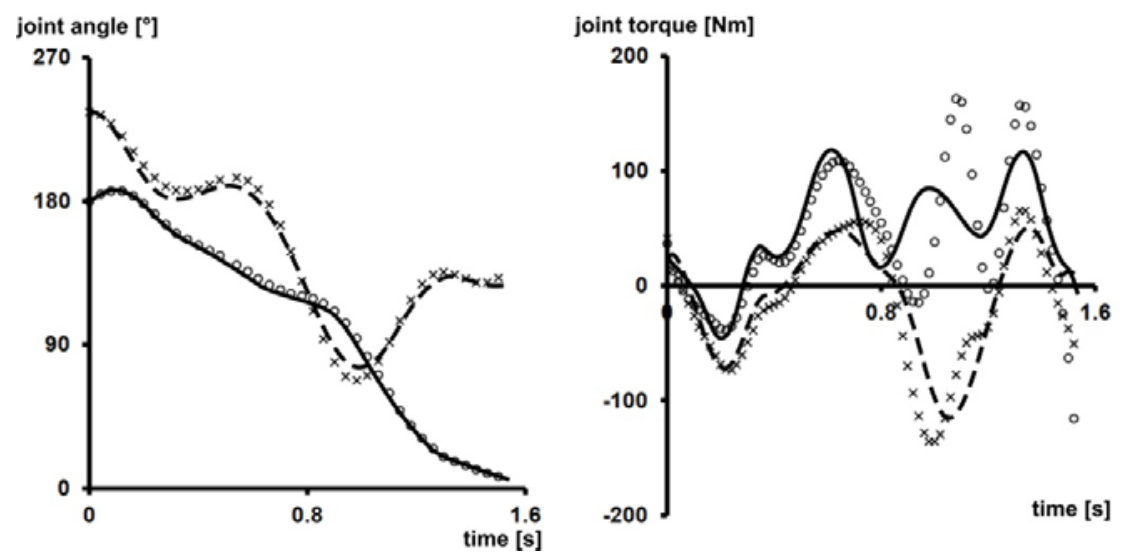

Figure 5. Joint angle and joint torque time histories of optimal solutions (solid and dashed lines) for: (a) minimising torque, (b) minimising torque change and (c) maximising success, compared with the matching simulation (circles and crosses).

\section{DISCUSSION}

Computer simulation is a useful tool that allows the researcher to investigate the underlying mechanics of human movement. It can also be used to investigate the control strategy being adopted by an athlete through the use of optimisation techniques. In the present study optimisation criteria based on minimising joint torques were compared with a criterion based on ensuring successful completion of the desired skill. The upstart is recognised in the coaching literature as requiring good "timing" in order to complete the skill successfully. That is, the gymnast has to co-ordinate her movements in time with the swing. This means that the gymnast has to perform the correct actions at the correct 
time in order to perform the upstart successfully. Given that human movement will always contain some level of variability (Bartlett, Wheat and Robins, 2007) it is not surprising that, when kinematic variability is included in the optimisation, a criterion based on maximising success produces a technique close to the recorded performance.

(a)

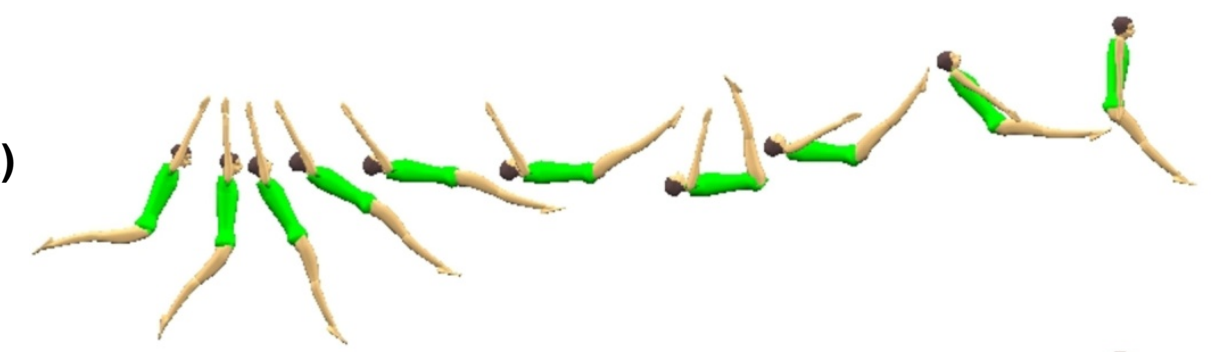

(b)

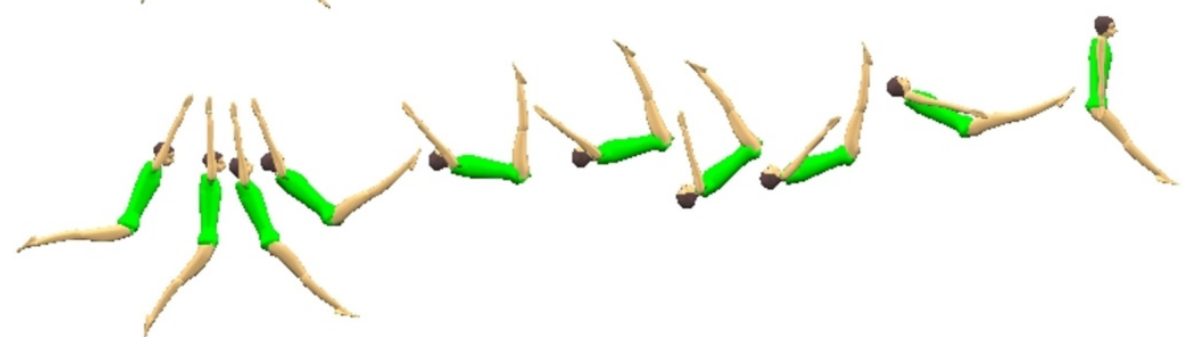

(c)

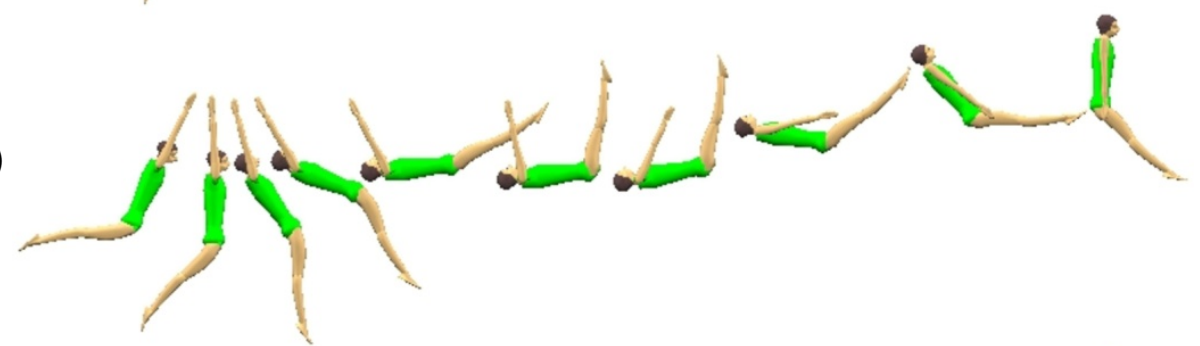

(d)

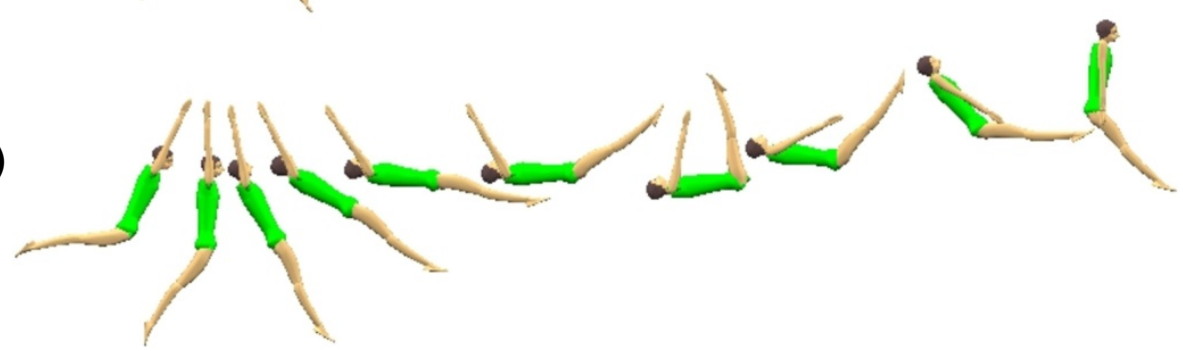

$\begin{array}{lllll}1 & 2 & 3 & 4 & 5\end{array}$

6

7

8

$9 \quad 10$

Figure 6. Graphics sequences of: (a) the matching simulation of the recorded performance and the optimal solutions for (b) minimising torque, (c) minimising torque change and (d) maximising success.

Previous research has shown that minimising joint torque may explain the technique used by gymnasts (Hiley and Yeadon, 2012a), although, in that example there was more than one recognised technique for producing the movement. The technique Hiley and Yeadon (2012a) predicted by minimising torque had a specific use in the development of the undersomersault: that is the technique is used when the gymnasts are young and do not have the strength to use the stoop stalder technique adopted by senior gymnasts. The technique used by the senior gymnasts required close to maximum effort in order to produce the desired movement outcome and could not be explained by minimising joint torques. In the case of the upstart, minimising joint torque or torque change produced techniques unlike those used by gymnasts (Figure 6 and Yamasaki et al., 2010). In other 
words, the upstart technique used by gymnasts is not a result of trying to minimise joint torque. It is noted that the optimal solutions based on minimising joint torque and torque change from the present study (Figure $6 \mathrm{~b}$ and c) display similar characteristics to the corresponding solutions obtained without via points by Yamasaki et al. (2010), particularly around the point of reversal in the direction of the swing (Figure $6 \mathrm{~b}$ and $\mathrm{c}$, graphic 7).

Yamasaki et al. (2010) describe the upstart as a "goal-directed" movement, where the goal is to successfully perform the skill (i.e. transfer the gymnast from below to above the bars and arrive in a predetermined configuration). It is therefore not surprising that technique is based on a criterion associated with such a classification: i.e. to successfully carry out the task each time it is attempted. In goal-directed tasks Wolpert (2007) described how technique is likely to be based on the probability of success given the variability inherent in the motor system. In other words, technique is learned, or evolves, so as to maximise success despite the limiting constraints of the noise within the system (Harris and Wolpert, 1998). If there were no movement variability in the human motor system and gymnasts could precisely recreate the same movement each time then technique could well be based on some criterion other than ensuring success. However, by the very nature of optimal solutions the introduction of a perturbation leads to a less than optimal performance (Hiley and Yeadon, 2008) and therefore a gymnast needs a technique that consistently produces success.

The third optimisation which aimed to maximise success was successful $100 \%$ of the time when perturbed, which may be argued is exactly what would be expected from the optimisation and of an elite gymnast. There were some small changes in technique compared to the recorded performance (Figures 5 and 6, Table 1), which may have been due to the method of determining the joint torque limits. The strength characteristics of the model were determined by scaling based on a matching simulation and it is unlikely that the gymnast in the video analysis was working at her strength limit during this particular upstart. This would have lead to an underestimation of the model's strength. Therefore, determining the maximal joint torques from a single performance is always likely to underestimate the true strength of the gymnast. Having a greater strength level may have resulted in the optimum technique (maximising success) being closer to the recorded performance.

The study used measures of kinematic variability obtained from swinging on bars (Hiley and Yeadon, 2012b). Cohen and Sternad (2009) used a decomposition of movement variability into components of noise, tolerance (how far the movements were from the successful solution space) and covariation (variation that was compensated for by redundancy) in relation to the task outcome. As it was not possible to break the variability down into its components and since the present simulation model was not capable of making feedback corrections, the assumption was made that only the noise component of variability was being considered. By using the average kinematic variability across a number of flexion and extensions it is possible that the noise component of the variability has been over-estimated, as the measured kinematic variability will probably include some feedback corrections.

The origin of the noise component of the kinematic variability is likely to be a result of noise within the neural system (Harris and Wolpert, 1998), therefore it could be argued that a muscle or torque driven simulation model would have been more appropriate to address the current problem. However, by using an angle driven model the variability added to the simulation could be based on measured kinematic variability, whereas 
determining the level of noise in the many components of the neural system would have been more problematic.

Movement variability is often described as the flexibility in the movement pattern which permits adaptation to perturbations or changing conditions. In this sense the variability is described as having a functional role. However, from the results of the present study it can be argued that the gymnast adopts a technique that lies within a solution space that is able to cope with the resultant noise from the motor system (Cohen and Sternad, 2009; Wolpert 2007). In other words the solution space surrounding the chosen technique is relatively insensitive to the inherent kinematic variability. Although both arguments can be used to describe the same situation the latter defines the source of the movement variability and how it relates to the successful outcome of the movement.

Trying to determine the underlying strategy using a single optimisation criterion may never be able to provide the whole story. Maximising success, in reality, finds the solution space which satisfies the constraints placed on the system. If the task is simple, that is, it only has a small number of constraints, the solution space may be large (i.e. constant success can be achieved with a number of techniques) and so the preferred technique might well be to adopt a minimal effort or energy strategy (e.g. walking, Ren, Jones and Howard, 2007). However, if the task is more complex, like the upstart (where the gymnast must remain within strength and anatomical limits, must arrive in a predetermined orientation and configuration and where the gymnast must perform correct actions at a correct time despite the presence of noise in the motor system) it might be that the solution space is so small that it effectively defines the technique.

\section{CONCLUSION}

Human movement variability has been described as having a functional role (Hamill, van Emmerick, Heiderscheit and Li, 1999; van Emmerick, Hamill, and McDermott, 2005; Bartlett et al., 2007), but it has also been described as having a component of "noise" (Wolpert, 2007; Cohen and Sternad, 2009) and as being due to redundancy in the sensorimotor system (Newell and Corcos, 1993). As variability will always be present in human movement (Bartlett et al., 2007) it will have an important influence on the success of a given task and therefore the technique adopted by athletes. Failing to consider such an important aspect can lead to optimal solutions that are not representative of human technique, resulting in incorrect conclusions. From the present study the underlying strategy used in the upstart is not based on a minimisation of joint torque: rather, it is likely to be based on a much more straight-forward strategy of ensuring success in the task despite operating in a "noisy" environment. In other words, gymnasts presumably develop and use techniques that are able to cope with the level of variability present in their own movements.

\section{Conflict of interest statement}

The authors wish to disclose that they have no financial or personal relationships with any people or organisations that could inappropriately influence this work. 


\section{REFERENCES}

Abdel-Aziz, Y.I., and Karara, H.M. (1971). Direct linear transformation from comparator coordinates into object space coordinates in close-range photogrammetry. ASP Symposium on Close-Range Photogrammetry (pp. 1-18). Falls Church, VA: American Society of Photogrammetry.

Bartlett, R., Wheat, J. and Robins, M. (2007). Is movement variability important for sports biomechanists? Sports Biomechanics, 6, 224-243.

Begon, M., Wieber, P-B. \& Yeadon, M.R. (2008). Kinematics estimation of straddled movements on high bar from a limited number of skin markers using a chain model. Journal of Biomechanics, 41, 581-586.

Broderick, M.P. and Newell, K.M. (1999). Coordination patterns in ball bouncing as a function of skill. Journal of Motor Behaviour, 31, 165-188.

Carroll, D.L. (2001). FORTRAN genetic algorithm driver. Downloaded from: http://cuaerospace.com/carroll.ga.html

Cohen, R.G. and Sternad, D. (2009). Variability in motor learning: relocating, channeling and reducing noise. Experimental Brain Research, 193, 69-83.

Davis, J. (2005). Undersomersaults on parallel bars. Gym Craft, 14, 6-7.

Fédération Internationale de Gymnastique. (2009). Code of Points. Moutier, Switzerland: F.I.G.

Forrester, S.E., Yeadon, M.R., King, M.A. and Pain, M.T.G. (2011). Comparing different approaches for determining joint torque parameters from isovelocity dynamometer measurements. Journal of Biomechanics, 44, 955-961.

Hamill, J., van Emmerick, R.E.A., Heiderscheit, B.C. and Li, L. (1999). A dynamical systems approach to lower extremity running injuries. Clinical Biomechanics, 14, 297308.

Harris, C.M. and Wolpert, D.M. (1998). Signal-dependent noise determines motor planning. Nature, 95, 780-784.

Hiley, M.J. and Yeadon, M.R. (2003). Optimum technique for generating angular momentum in accelerated backward giant circles prior to a dismount. Journal of Applied Biomechanics, 19, 119-130.

Hiley, M.J. and Yeadon, M.R. (2007). Optimisation of backward giant circle technique on the asymmetric bars. Journal of Applied Biomechanics, 23, 301-309.

Hiley, M.J. and Yeadon, M.R. (2008). Optimisation of high bar circling technique for consistent performance of a triple piked somersault dismount. Journal of Biomechanics, 41, 1730-1735.

Hiley, M.J. and Yeadon, M.R. (2012a). The effect of cost function on optimum technique of the undersomersault on parallel bars. Journal of Applied Biomechanics, 28, 10-19.

Hiley, M.J. and Yeadon, M. (2012b). Achieving consistent performance in a complex whole body movement: the Tkatchev on high bar. Human Movement Science, in press.

King, M.A., Kong, P.W. and Yeadon, M.R. (2009). Determining effective subject-specific strength levels for forward dives using computer simulation of recorded performances. Journal of Biomechanics, 42, 2672-2677. 
Newell, K.M. and Corcos, D.M. (1993). Variability and Motor Control. Human Kinetics. Champaign, IL.

Ren, L., Jones, R.K. and Howard, D. (2007). Predictive modelling of human walking over a complete gait cycle. Journal of Biomechanics, 40, 1567-1574.

van Emmerik, R.E.A., Hamill, J. and McDermott, W.J. (2005). Variability and coordinative function in human gait. Quest, 57, 102-123.

Wolpert, D.M. (2007). Probabilistic models in human sensorimotor control. Human Movement Science, 26, 551-524.

Wood, G.A. and Jennings, L.S. (1979). On the use of spline functions for data smoothing. Journal of Biomechanics, 12, 477-479.

Yamasaki, T., Gotoh, K. and Xin, X. (2010). Optimality of a kip performance on the high bar: An example of skilled goal-directed whole body movement. Human Movement Science, 29, 464-482.

Yeadon, M.R. (1990b). The simulation of aerial movement - I. The determination of orientation angles from film data. Journal of Biomechanics, 23, 59-66.

Yeadon, M.R. (1990a). The simulation of aerial movement - II. A mathematical inertia model of the human body. Journal of Biomechanics, 23, 67-74.

Yeadon, M.R. and King, M.A. (1999). A method for synchronising digitised video data. Journal of Biomechanics, 32, 983-986. 